\title{
A SURVEY FOR OUTER SATELLITES OF MARS: LIMITS TO COMPLETENESS
}

\author{
Scott S. Sheppard, ${ }^{1}$ David Jewitt, and Jan Kleyna \\ Institute for Astronomy, University of Hawaii, 2680 Woodlawn Drive, Honolulu, HI 96822; \\ sheppard@dtm.ciw.edu, jewitt@ifa.hawaii.edu,kleyna@ifa.hawaii.edu \\ Received 2004 June 28; accepted 2004 July 15
}

\begin{abstract}
We have surveyed the Hill sphere of Mars for irregular satellites. Our search covered nearly the entire Hill sphere, but scattered light from Mars excluded the inner few arcminutes where the satellites Phobos and Deimos reside. No new satellites were found to an apparent limiting red magnitude of 23.5, which corresponds to radii of about $0.09 \mathrm{~km}$ using an albedo of 0.07 .

Key words: planets and satellites: general — planets and satellites: individual (Mars)
\end{abstract}

\section{INTRODUCTION}

The planets have two main types of satellites (Peale 1999). Regular satellites have nearly circular, low-inclination, prograde orbits. These satellites probably formed within a disk of gas and dust around the planet, analogous to the disk from which the planets formed around the Sun. Irregular satellites have large, eccentric orbits that are usually highly inclined and even retrograde relative to the equatorial plane of their planet. These irregular satellites cannot have formed by circumplanetary accretion. Instead, they were probably captured from heliocentric orbit during the epoch of formation of the planets (Kuiper 1956; Pollack et al. 1979).

Permanent satellites are confined to the region of gravitational influence, known as the Hill sphere. The radius of the Hill sphere is

$$
r_{\mathrm{H}}=a_{p}\left(\frac{m_{p}}{3 M_{\odot}}\right)^{1 / 3}
$$

where $a_{p}$ and $m_{p}$ are the semimajor axis and mass of the planet and $M_{\odot}$ is the mass of the Sun. From equation (1) and using a mass for Mars of $0.64 \times 10^{24} \mathrm{~kg}$, a solar mass of $1.99 \times 10^{30} \mathrm{~kg}$, and data from Table 1, we find that Mars's Hill sphere is about 0.71 in radius, which corresponds to about 1.6 square degrees in area as seen from Earth during our observations.

The availability of sensitive, large-scale CCD detectors has refreshed the study of irregular satellites by enabling a new wave of discovery around Jupiter (Sheppard \& Jewitt 2003), Saturn (Gladman et al. 2001), Uranus (Gladman et al. 2000), and Neptune (Holman et al. 2003; Sheppard et al. 2003). Large fields of view are needed because the Hill spheres are large. Sensitivity is needed because the majority of irregular satellites are small and therefore faint. From these new surveys it appears that the four giant planets possess about the same number of irregular satellites and groupings, with no dependence on the planet's mass when comparing them with the same limiting size of satellites (Sheppard \& Jewitt 2003). For an indepth review of irregular satellites, see Jewitt et al. (2004).

Mars is known to have two small inner satellites, Phobos and Deimos (Hall 1878). The origins of these satellites are still a mystery. Their orbits are not like those of the irregular satellites

\footnotetext{
${ }^{1}$ Current address: Department of Terrestrial Magnetism, Carnegie Institution of Washington, 5241 Broad Branch Road, NW, Washington, DC 20015.
}

of the giant planets, since they are close to Mars and have nearly zero eccentricity and inclination. Mars's satellites, from a dynamical perspective, appear to have originated not far from their current positions, because it is hard to produce orbits like those of the known Martian satellites by capture. On the other hand, the physical properties of Phobos and Deimos resemble those of C-type asteroids, most of which are found in the outer main belt, not near Mars. Based on their physical characteristics, they may be captured asteroids. In addition, the current orbit of Phobos is short-lived and the satellite will collide with the planet on a timescale of $\sim 10^{8}$ yr (see Burns 1992 and references therein).

Kuiper (1961) briefly reviewed some early photographic searches for outer satellites of Mars. These surveys only reached a photographic magnitude of about 17 (about $1.4 \mathrm{~km}$ in diameter, assuming an albedo of 0.05). The Viking Orbiter 1 spacecraft found no new satellites of Mars larger than about $0.05 \mathrm{~km}$ within the orbit of Phobos (Duxbury \& Ocampo 1988). Showalter et al. (2001) used the Hubble Space Telescope (HST) to search for possible Mars dust rings created by Phobos and Deimos that had been predicted by Soter (1971). They did not detect any dust rings or unknown satellites down to about $0.1 \mathrm{~km}$ in diameter (assuming an albedo of 0.07 ) in the very inner portions of Mars space near Phobos and Deimos. The $H S T$ observations have not been published, but the small field of view (a few arcminutes) in any case allowed them to survey only the very inner portion of the Hill sphere of Mars. In this sense, our survey is complementary to theirs.

Only the giant planets have been found to possess abundant irregular satellites. Are the distant irregular satellites unique to the giant planets or does the outermost terrestrial planet, Mars, have them as well? Like Jupiter and Neptune, Mars has Trojan asteroids in its leading and trailing Lagrangian regions, which might have been captured in a similar way to irregular satellites (Marzari et al. 2002). With modern wide-field digital detectors on medium-class telescopes, we can now survey the space around Mars to much fainter magnitudes than the early photographic surveys. In this paper, we describe such a survey in which we used a wide-field digital detector to perform a search of the Martian Hill sphere for satellites several magnitudes fainter than previously achieved.

\section{OBSERVATIONS}

We used the $3.6 \mathrm{~m}$ diameter Canada-France-Hawaii Telescope atop Mauna Kea in Hawaii with the MegaCam digital 
TABLE 1

Geometric Circumstances

\begin{tabular}{cccccc}
\hline \hline $\begin{array}{c}\text { UT } \\
\text { Date }\end{array}$ & $\begin{array}{c}R \\
(\mathrm{AU})\end{array}$ & $\begin{array}{c}\Delta \\
(\mathrm{AU})\end{array}$ & $\begin{array}{c}\alpha \\
(\mathrm{deg})\end{array}$ & $\begin{array}{c}\text { R.A. } \\
\left(\operatorname{arcsec~hr}^{-1}\right)\end{array}$ & $\begin{array}{c}\text { Decl. } \\
\left(\operatorname{arcsec~hr}^{-1}\right)\end{array}$ \\
\hline 2003 Jul 5 ..... & 1.403 & 0.536 & 35.68 & 38.1 & 7.2 \\
2003 Jul 7 ..... & 1.401 & 0.525 & 35.00 & 35.4 & 6.2 \\
\hline
\end{tabular}

CCD camera for imaging (Boulade et al. 2003). MegaCam has 36 separate CCDs, with each having $2048 \times 4612(13.5 \mu \mathrm{m})$ pixels, which at prime focus gives $0{ }^{\prime \prime} 187 \mathrm{pixel}^{-1}$ scale. The total number of pixels is about 340 million and each image was about 710 megabytes in size. The field of view is $0.96 \times$ 0.94 with gaps of about $13^{\prime \prime}$ between most chips (though a few have gaps of about $80^{\prime \prime}$ ). MegaCam uses a wide-field corrector in order to produce uniform image quality across the field of view.

Images were obtained in queue scheduling mode by staff observers. An $r^{\prime}$ filter based on the Sloan Digital Sky Survey filter set was used. The images were bias-subtracted and then flat-fielded using twilight flats. During exposures the telescope was autoguided sidereally on bright nearby stars. The nights were photometric, and the seeing was about 0 "'7 during the observations. Integration times were $100 \mathrm{~s}$ on July 5 and $120 \mathrm{~s}$ on July 7 . The observing geometry and characteristics of Mars at the time of the observations are shown in Table 1.

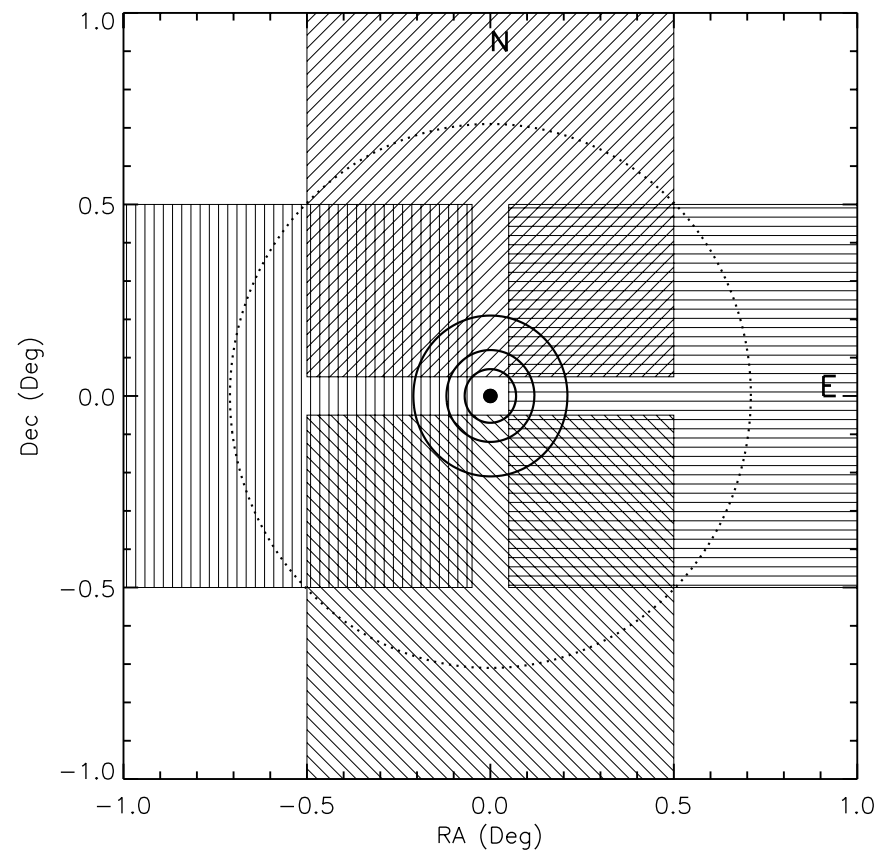

FIG. 1.- Area searched around Mars for satellites using the Canada-FranceHawaii Telescope. Four fields were imaged three times each on two separate nights (UT 2003 July 5 and 7) for a total of 24 images. The black dot at the center represents Mars's position. The dot is larger than the apparent diameter of Mars and incorporates the orbits of Phobos and Deimos. The four different hatched squares represent the four fields imaged around Mars on both nights. Overlapping hatches show where some of the fields overlapped in coverage. The dotted circle shows the Hill sphere of Mars to our line of sight. The scattered light from Mars was not a problem for most of the images but became quickly dominant very near Mars. The inner solid circles show where scattered light became significant compared with the normal sky background. At the outer solid circle, near $0^{\circ} 20$, the background was about 1.5 times higher, while at the middle solid circle the background was about 2 times higher and at the inner solid circle the background was about 3.75 times higher than the nominal sky background.

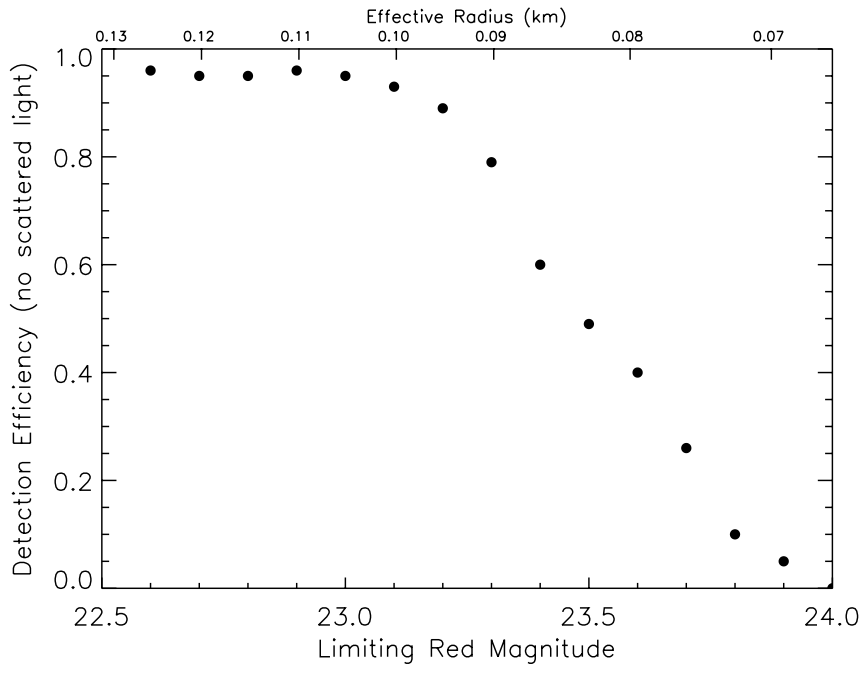

FIG. 2.-Detection efficiency of the moving-object search computer program vs. the apparent red magnitude. The $50 \%$ differential detection efficiency is at about $23.5 \mathrm{mag}$. The differential efficiency was determined by how many artificially generated moving objects with motions similar to that of Mars were found by the program at a given magnitude. Magnitude bins were separated by 0.1 . This efficiency is for no significant scattered light in the fields. The calculation of the effective radius assumes an albedo of 0.07 .

Figure 1 shows the area searched around Mars for possible satellites. The entire outer Martian Hill sphere was covered on each night of UT 2003 July 5 and 7. Four fields were imaged three times each on both nights, for a total of 24 images. On a given night, the images of each field were separated by about 13.5 minutes, giving 27 minutes between the first and last images. A computer program was used to identify objects that were detected in all three images from one night and had a motion within $\pm 6^{\prime \prime} \mathrm{hr}^{-1}$ of Mars's motion in both right ascension and declination. This motion is consistent with parallactic displacement of an object moving near Mars. There was no confusion with main-belt asteroids, since they tend to stay away from Mars's orbit and Mars was near perihelion during the observations.

Each night was photometric, and Landolt (1992) standards were used to calibrate the data. The apparent red limiting magnitude of the survey was found by placing artificially generated objects with motions similar to that of Mars into the survey fields. The artificial objects had a range of magnitudes and were matched to the point-spread function of the images in the north-south direction (FWHM $\sim 0.7$ ) and were elongated in the east-west direction to simulate the slight trailing any real satellites would have incurred ( $\sim 1$ "' 1$)$ during the exposure. The $50 \%$ differential detection efficiency for artificial moving objects in the survey fields was found at an $R$-band magnitude of about 23.5. Figure 2 shows the differential detection efficiency of the search program versus limiting red magnitude. The multiple nights of observations, which would only help increase our detection rate, were not factored into the efficiency.

The above results apply when no significant amount of scattered light is present. Figures 1 and 3 show the behavior of the scattered light near Mars. Scattered light was noticed up to about $20^{\prime}$ from Mars. Figure 4 shows the $50 \% R$-band limiting magnitude of the program versus the distance from Mars.

\section{RESULTS AND DISCUSSION}

We found no new satellites of Mars to a limiting apparent red magnitude of 23.5. In order to determine the corresponding 


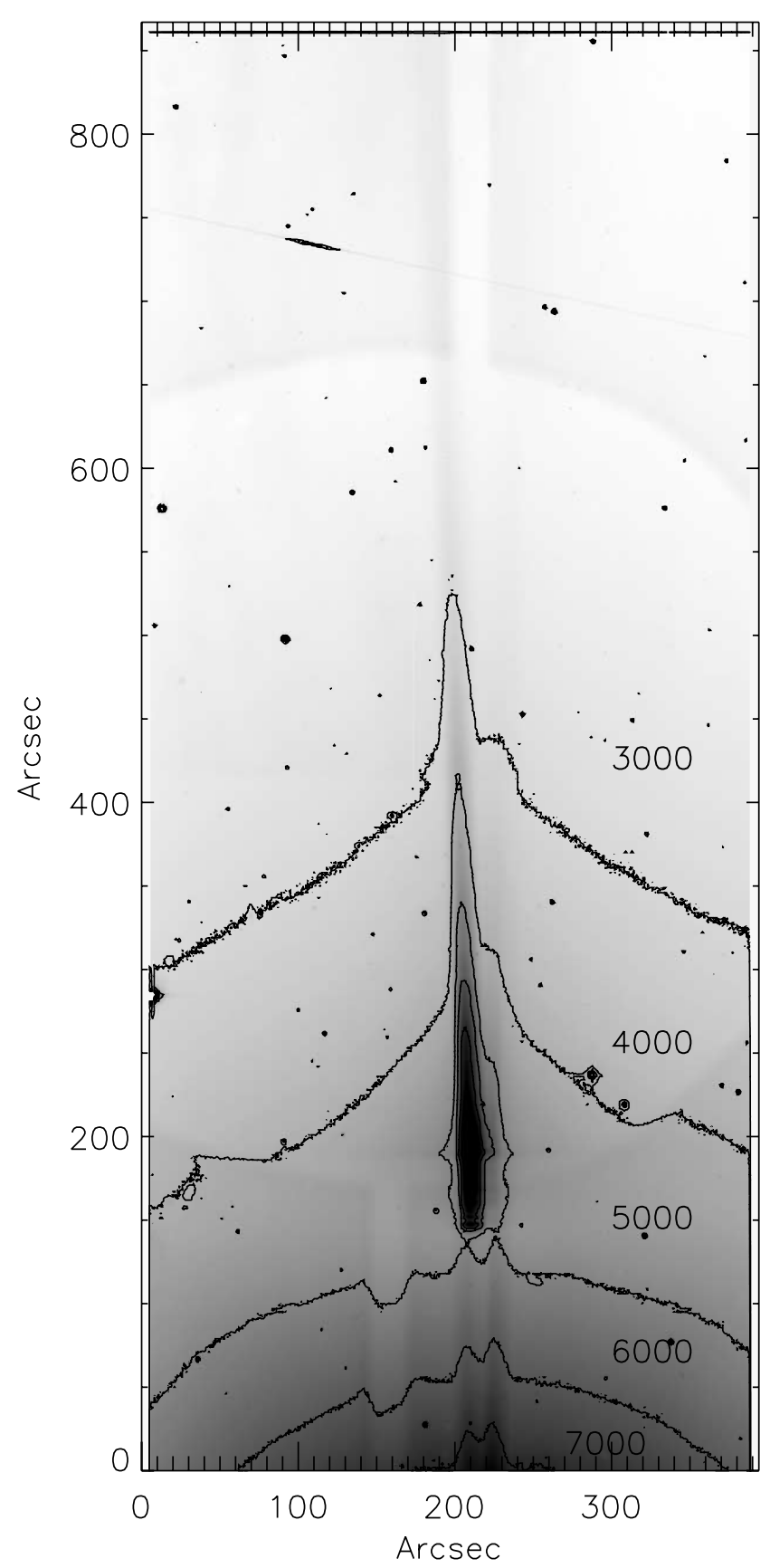

FIg. 3.-Contour plot of a survey image close to Mars to show the scattered light. The contours show $7000: 6000: 5000: 4000: 3000$ surface brightness contours, where the nominal background sky level away from the planet was near 2000 counts. Mars is about $3^{\prime}$ south from the bottom of the plotted region. The vertical spike is caused by diffraction from the telescope's support structure. The image color map has been inverted so that higher counts are darker. The color map was also scaled logarithmically to bring out more detail between the top and bottom of the image.

size limit, we relate the apparent red magnitude of an object, $m_{R}$, to its radius, $r$, through

$$
r=\left[\frac{2.25 \times 10^{16} R^{2} \Delta^{2}}{p_{R} \phi(\alpha)}\right]^{1 / 2} 10^{0.2\left(m_{\odot}-m_{R}\right)},
$$

in which $r$ is in kilometers, $R$ is the heliocentric distance in $\mathrm{AU}, \Delta$ is the geocentric distance in $\mathrm{AU}, m_{\odot}$ is the apparent red magnitude of the Sun $(-27.1), p_{R}$ is the geometric red albedo, and $\phi(\alpha)$ is the phase function, in which the phase angle

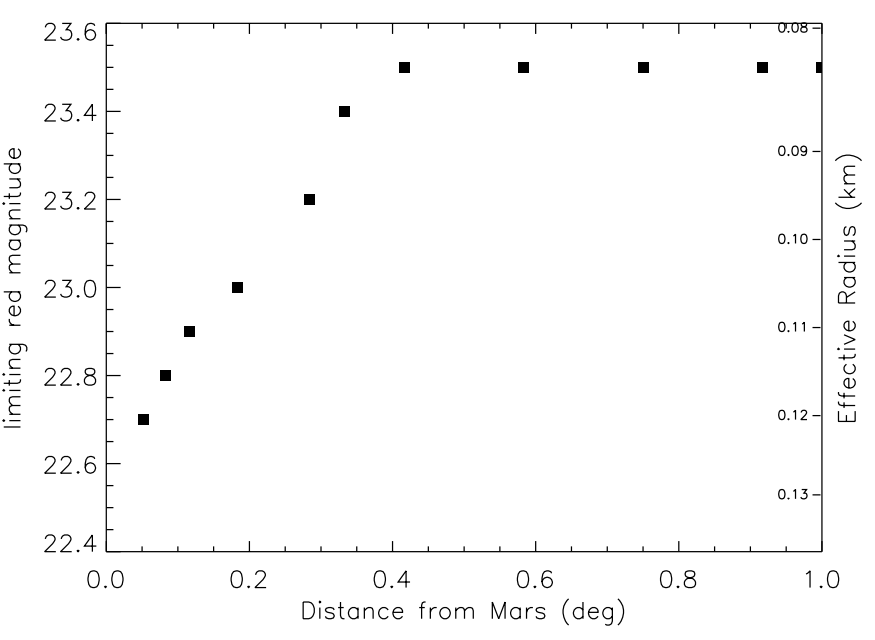

FIG. 4. - The 50\% efficiency detection red limiting magnitude of the survey vs. distance from Mars. Scattered light is only significant starting at about $15^{\prime}$ from Mars. The calculation of the effective radius assumes an albedo of 0.07.

$\alpha=0^{\circ}$ at opposition. For linear phase functions we use the notation $\phi(\alpha)=10^{-0.4 \beta \alpha}$, where $\beta$ is the "linear" phase coefficient. If we use the data from Table 1 and a phase coefficient of $\beta=0.05 \mathrm{mag} \mathrm{deg}^{-1}$ and assume the albedo is 0.07 , we find that an apparent magnitude of 23.5 corresponds to objects that are about $0.09 \mathrm{~km}$ in radius at Mars's distance. With this sensitivity we would have easily detected any Phobos $(r \sim 11 \mathrm{~km})$ or Deimos $(r \sim 6 \mathrm{~km})$ sized satellites in our fields. The survey covered $99.5 \%$ of Mars's Hill sphere. Only the inner $\sim 3^{\prime}\left(\leq 0.07 r_{\mathrm{H}}\right)$ near Mars was not covered in our survey, because of scattered light from the planet.

Mars's lack of outer satellites compared with the giant planets is shown in Figure 5 and Table 2. By comparing Mars's Hill sphere with the giant planets', we would expect to find outer irregular satellites much more distant than Phobos and Deimos. Why are there no known irregular satellites around the terrestrial planets? Previous authors have found that the inner terrestrial planets Mercury and Venus probably have no satellites as a consequence of strong solar tides, which would make them unstable (Counselman 1973; Ward \& Reid 1973; Burns 1973). Earth and Mars are spared extreme solar tides. Earth might have lost small outer satellites because of the presence of the large Moon (Callegari \& Yokoyama 2001).

There are two basic reasons why Mars may have no irregular satellites. Either such objects are dynamically unstable on timescales comparable to or less than the age of the solar system, or Mars lacked any mechanism to capture irregular satellites in the first place. The proximity of Mars to Jupiter may limit distant stable orbits about Mars. The role of these perturbations on long timescales and as a function of location within Mars's Hill sphere is still yet to be fully explored. Analysis of main-belt asteroid satellite stability shows that the Sun is the dominant perturber for asteroids in the mid-to-inner main belt, while Jovian perturbations become dominant in the outer main belt (Chauvineau \& Mignard 1990). Extrapolation to Mars suggests that satellites could be stable over a significant portion of the Hill sphere for the age of the solar system.

Alternatively, the deficiency of irregular satellites around Mars may arise from the difference in the formation process of Mars compared with the giant planets. Irregular-satellite capture for the giant planets probably occurred toward the end of each planet's formation epoch, when the energy dissipation needed for capture could be attributed to gas drag from an 


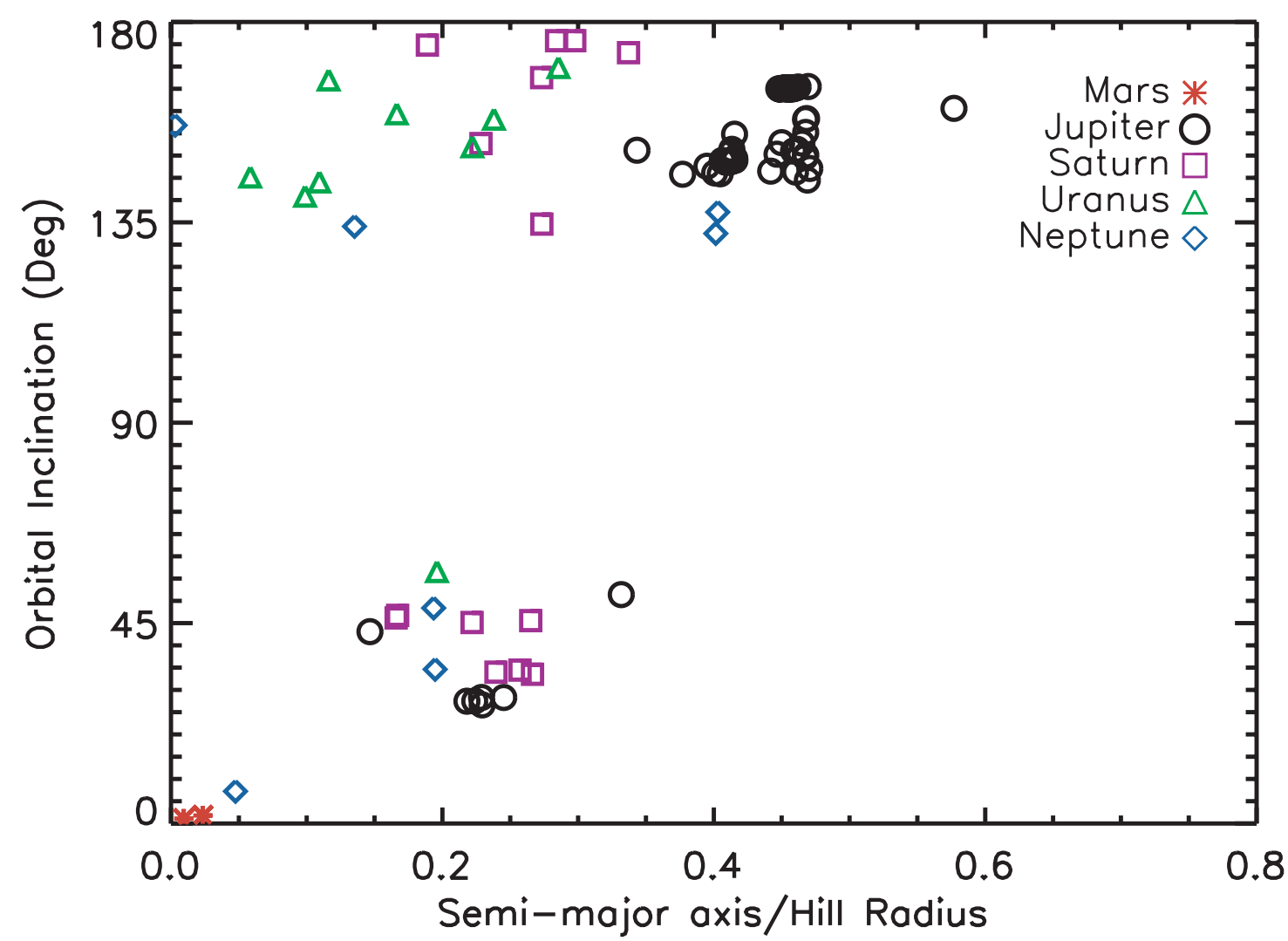

Fig. 5. - Comparison of the known irregular satellites of the giant planets with Mars's two known satellites. The horizontal axis is the ratio of the satellite's semimajor axis to the respective planet's Hill radius. The vertical axis is the inclination of the satellite to the ecliptic plane. Only Nereid, of Neptune, is close to the position of the known Martian satellites in this parameter space.

extended atmosphere, the enlargement of the Hill sphere caused by the planet's mass growth, or higher collision probabilities with nearby small bodies (Kuiper 1956; Colombo \& Franklin 1971; Heppenheimer \& Porco 1977; Pollack et al. 1979). Unlike the giant planets, Mars is not expected to have

TABLE 2

Irregular Satellites of the Planets

\begin{tabular}{crcccc}
\hline \hline Planet & Irr. $^{\mathrm{a}}$ & Groups $^{\mathrm{b}}$ & $\begin{array}{c}r_{\text {min }} \text { Limit }^{\mathrm{c}} \\
(\mathrm{km})\end{array}$ & $\begin{array}{c}\text { Hill Radius } \\
(\mathrm{deg})\end{array}$ & $\begin{array}{c}\text { Hill Area }^{\mathrm{e}} \\
(\mathrm{deg})\end{array}$ \\
\hline Mars .............. & 0 & 0 & 0.09 & 0.7 & 1.6 \\
Jupiter........... & 55 & $\sim 6$ & 1 & 4.7 & 70 \\
Saturn ............ & 14 & $\sim 4-5$ & 4 & 3.0 & 28 \\
Uranus .......... & 9 & $\sim 2-3$ & 7 & 1.4 & 6 \\
Neptune .......... & 7 & $\sim 3-4$ & 16 & 1.5 & 7 \\
\hline
\end{tabular}

${ }^{\text {a }}$ Number of known irregular satellites.

b Apparent number of dynamical groupings.

${ }^{c}$ Approximate limiting radii of satellite searches to date, assuming low albedos.

${ }^{\mathrm{d}}$ The apparent angular Hill sphere radius of the planet at opposition.

e The Hill sphere area of a planet when at opposition. gone through a significant Hill sphere enlargement from a large mass accretion event or to have had a dense, extended atmosphere during formation.

\section{CONCLUSIONS}

No outer satellites of Mars larger than about $0.09 \mathrm{~km}$ in radius were detected in our survey, which covered $99.5 \%$ of Mars's Hill sphere (from $3^{\prime}$ to over 0.7 from the planet). The absence of outer satellites around Mars may indicate that it did not have the right conditions for irregular-satellite capture as did the giant planets during their formation, may show that such objects are dynamically unstable, or both.

We thank Jing Li for help on IDL contour plotting, Francesco Marzari for a prompt review, and the CFHT queue service observing team for proficient data handling. This work was supported by a grant to D. J. from NASA. The Canada-FranceHawaii Telescope is operated by the National Research Council of Canada, the Centre National de la Recherche Scientifique of France, and the University of Hawaii.
Boulade, O., et al. 2003, Proc. SPIE, 4841, 72

Burns, J. A. 1973, Nature Phys. Sci., 242, 23 1992, in Mars, ed. H. H. Kieffer, B. M. Jakosky, C. Snyder, \&

M. S. Matthews (Tucson: Univ. Arizona Press), 1283

Callegari, N., Jr., \& Yokoyama, T. 2001, Planet. Space Sci., 49, 35

Chauvineau, B., \& Mignard, F. 1990, Icarus, 87, 377

Colombo, G., \& Franklin, F. A. 1971, Icarus, 15, 186

\section{REFERENCES}

Counselman, C. C., III. 1973, ApJ, 180, 307

Duxbury, T. C., \& Ocampo, A. C. 1988, Icarus, 76, 160

Gladman, B., et al. 2001, Nature, 412, 163

Gladman, B., Kavelaars, J. J., Holman, M., Petit, J.-M., Scholl, H., Nicholson, P., \& Burns, J. A. 2000, Icarus, 147, 320 (erratum 148, 320)

Hall, A. 1878, Astron. Nachr., 92, 47

Heppenheimer, T. A., \& Porco, C. 1977, Icarus, 30, 385 
Holman, M., et al. 2003, IAU Circ. 8047

Jewitt, D. C., Sheppard, S., \& Porco, C. 2004, in Jupiter: The Planet, Satellites and Magnetosphere, ed. F. Bagenal, T. Dowling, \& W. McKinnon (Cambridge: Cambridge Univ. Press), chap. 12

Kuiper, G. P. 1956, Vistas Astron., 2, 1631

1961, in Planets and Satellites, ed. G. P. Kuiper \& B. M. Middlehurst (Chicago: Univ. Chicago Press), 575

Landolt, A. U. 1992, AJ, 104, 340

Marzari, F., Scholl, H., Murray, C., \& Lagerkvist, C. 2002, in Asteroids III, ed. W. F. Bottke, A. Cellino, P. Paolicchi, \& R. P. Binzel (Tucson: Univ. Arizona Press), 725
Peale, S. J. 1999, ARA\&A, 37, 533

Pollack, J. B., Burns, J. A., \& Tauber, M. E. 1979, Icarus, 37, 587

Sheppard, S. S., \& Jewitt, D. C. 2003, Nature, 423, 261

Sheppard, S. S., et al. 2003, IAU Circ. 8193

Showalter, M. R., Hamilton, D. P., \& Nicholson, P. D. 2001, BAAS, 33, 1095

Soter, S. 1971, The Dust Belts of Mars (Cent. Radiophys. Space Res. Rep. 462) (Ithaca: Cornell Univ.)

Ward, W. R., \& Reid, M. J. 1973, MNRAS, 164, 21 